\title{
The prevalence of IgG and IgA against adenoviruses in serum of children aged 11-26 months, hospitalised in the Clinical Paediatric Hospital in Warsaw, Poland
}

\author{
ZUZANNA TROJNAR ${ }^{1}$, OLGA CIEPIELA2, URSZULA ANNA DEMKOW² \\ ${ }^{1}$ Students Scientific Group at Department of Laboratory Diagnostics and Clinical Immunology of Developmental Age, \\ Medical University of Warsaw, Poland \\ ${ }^{2}$ Department of Laboratory Diagnostics and Clinical Immunology of Developmental Age, Medical University of Warsaw, Poland
}

\begin{abstract}
Adenoviruses are widely spread viruses causing infections related mainly to children. Contact with the pathogen may result in the presence of either immunoglobulin (Ig) A or IgG antibodies in the serum. The aim of this study was to assess the prevalence of $\mathrm{IgA}$ and $\mathrm{Ig} G$ antibodies against adenoviruses in serum of children aged 11-27 months, hospitalised at the Public Children's Clinical Hospital in Warsaw. Immunoassay tests DRG Adenovirus IgA ELISA and IgG DRG Adenovirus ELISA detecting antibodies against all pathogenic serotypes of adenoviruses were performed in 128 sera samples. A low percentage of positive results for both immunoglobulins, 17 cases (13.28\%) for IgA and 16 cases (12.50\%) for IgG antibodies respectively were reported. We conclude, that the prevalence of antibodies against adenoviruses does not coincide with epidemiological data on the spread of infections among young children. The assays performed may be more useful in distinguishing the active form of the infection from seropositivity due to prior infection.
\end{abstract}

Key words: adenoviruses, antibodies, ELISA, IgA, IgG.

(Centr Eur J Immunol 2014; 39 (1): 91-95)

\section{Introduction}

Adenoviral infections constitute a significant proportion of all infections caused by viruses [1,2]. Adenoviruses are widely spread viruses causing mild infections in immunocompetent patients, while providing a serious risk to immunocompromised patients [3]. Infections caused by adenoviruses relate mainly to children; therefore, it is suggested that the infection develops a life-long term immunological memory. Recent infection may also results in the presence of immunoglobulin (Ig) A and IgG antibodies in the serum of people who come into contact with the adenovirus. According to Nadeau et al. approximately $50 \%$ of the human population has antibodies against adenoviruses, which clearly may indicate the widespread nature of infections caused by these pathogens $[4,5]$. The high frequency of seropositivity in the general population is associated with repeated infections with various forms of the virus in early childhood. Antibodies against endemic types of adenoviruses are acquired at an early age, and most children have had contact with various adenoviruses before 6 years of age [6]. The prevalence of antibodies may increase with age, most likely due to an increase frequency of exposure over a lifetime [7].

The presence of adenoviruses can be confirmed by analysing the presence of specific antibodies or isolation of viral strains. Because the infection is associated with production of specific IgM, IgA and IgG antibodies, studying their occurrence should give reliable information on the epidemiology of adenoviral infections [8].

Our aim was to assess the prevalence of $\operatorname{IgA}$ and $\mathrm{IgG}$ antibodies against adenoviruses in the serum of children hospitalised due to different diseases.

\section{Material and methods}

Residual serum samples were collected from 128 patients, 70 boys and 58 girls, aged eleven to twenty-seven months (median 19 months old) hospitalised at the Public Children's Hospital in Warsaw. Fifteen of the 128 patients were hospitalised due to diarrhoea and tests for adenoviral and rotaviral antigens in the faeces of these patients

Correspondence: Olga Ciepiela, PhD, Department of Laboratory Diagnostics and Clinical Immunology of Developmental Age, Medical University of Warsaw, Marszłkowska 24, 00-576 Warsaw, Poland, e-mail: olga.ciepiela@wum.edu.pl 
were performed. None of the children had a positive test result for adenovirus presence in faeces, but in two samples rotaviruses were detected. The enrolled patients were treated in different hospital departments, including surgery, endocrinology, haematology, laryngology, cardiology and infants. According to the rules of medical information confidentiality, we did not obtain information regarding reason for hospitalisation. Exclusion criteria consisted of humoral immunodeficiency and haematological or lymphatic disorders. Children suffering from lymphomas, leukemias and recurrent infection were excluded based on results of complete blood count, results of immunophenotyping of bone marrow, peripheral blood or lymph gland tissue and referral for chemiluminescence test. Children hospitalised for abscesses surgery were excluded based on referral for pus culture. Samples were stored at $-20^{\circ} \mathrm{C}$ and on the day of testing samples were thawed at room temperature. To perform both tests a total of $200 \mu \mathrm{l}$ of residual serum were used. Patients were also divided into two groups: 11-18 months old and 19-27 months old. In the first group there were 58 patients $(45.31 \%)$, including 28 girls (48.28\%) and 30 boys $(51.72 \%)$, and in the second group there were 70 patients $(54.69 \%)$, including 30 girls $(42.86 \%)$ and 40 boys $(57.14 \%)$.

For the qualitative detection of antibodies ( $\operatorname{IgA}$ and $\mathrm{IgG}$ ) against all pathogenic human adenovirus serotypes in the serum samples the DRG Adenovirus IgA ELISA (DGR International, Germany) and DRG Adenovirus IgG ELISA (DRG International, Germany) were performed. Specific antibodies were detected according to the manufacturer's instructions. Serum samples were diluted with sample diluent and additionally (for IgA detection) incubated with IgG-RF-Sorbent containing hyper-immune anti-human IgG-class antibody to eliminate competitive inhibition from specific $\mathrm{IgG}$ and to remove rheumatoid factors. Diluted samples were pipetted into wells and during incubation specific antibodies were bounded to the immobilised antigens. Unbounded conjugates were then removed by washing. During a second incubation anti-human (IgA or IgG) antibodies conjugated with horseradish peroxidase conjugate were bound to immobilised antibodies and formed enzyme-linked immune complexes. After second washing, unbounded complexes were removed. After adding substrate and stop solution, the change in colour to yellow indicated positive samples. The absorbance was determined for each well at $450 \mathrm{~nm}$ and reference wavelength at $620 \mathrm{~nm}$.

\section{Interpretation of ELISA results}

The ELISA results were interpreted according to the instructions of the manufacturer. Briefly, a cut-off (CO) value was obtained from mean absorbance value of the two cut-off control determinations. Sample with OD (optical density) of $>1.1 \times \mathrm{CO}$ was considered to be positive, the patient (mean) absorbance values from $10 \%$ above to $10 \%$ below $\mathrm{CO}$ were considered to be in the grey zone. Samples with absorbance more than $10 \%$ below $\mathrm{CO}$ were negative.

\section{Ethical consideration}

The study was approved by the Ethical Committee of Warsaw Medical University.

\section{Statistical analysis}

Data were analysed using the $\chi^{2}$ test and Fisher's exact test for small amounts on STATISTICA software (version 10). A $p$ value of $<0.05$ was considered significant.

\section{Results}

\section{The prevalence of $\operatorname{IgA}$ antibodies to adenoviruses}

In the study group $(n=128)$ positive results in IgA antibodies were obtained in 17 children $(13.28 \%)$ and the grey zone results in 2 patients $(1.56 \%)$ (Fig. 1).

In the age group of $11-18$ months $(n=58)$ positive results were obtained in 8 children $(13.79 \%)$. In the group of children aged 19-27 months $(n=70) 9(12.86 \%)$ positive results were obtained. There was no significant difference in the number of positive results between analysed groups $(p=0.5022)$.

In addition, in both age groups the frequency of positive and grey-zone results with regard to the gender of patients were assessed. In the group of 11-18-montholds, in which there were 28 girls and 30 boys, positive results were obtained in five girls $(17.86 \%)$ and three boys $(10.00 \%)$, while the positive results in the grey zone were obtained for one girl (3.57\%) and one boy (3.33\%). In the age group of 19-27 months, in which there were 30 girls and 40 boys, positive results were obtained for 3 girls $(10.00 \%)$ and 6 boys $(15.00 \%)$. There were no results in the grey zone for both girls or boys in this age group. No statistically significant difference between the positive groups of girls aged 11-18 months and 19-27 months $(p=0.4637)$, as well as between groups of boys aged 11-18 months and 19-27 months ( $p=0.7227)$, were noted.

\section{The prevalence of IgG antibodies to adenoviruses}

Immunoglobulin $\mathrm{G}$ antibodies against adenoviruses were detected in the serum from 16 children (12.50\%), and the results in the grey zone were obtained in five samples (3.91\%) (Fig. 2).

In the age group $11-18$ months $(n=58) 6$ positive results $(10.34 \%)$ were obtained as well as $2(3.45 \%)$ in the grey zone. In the age group $19-27$ months $(n=70)$ positive results were obtained in 10 children $(14.29 \%)$ and results in grey zone were seen in 3 samples (4.29\%). There was no statistically significant difference between analysed groups $(p=0.5022)$. 


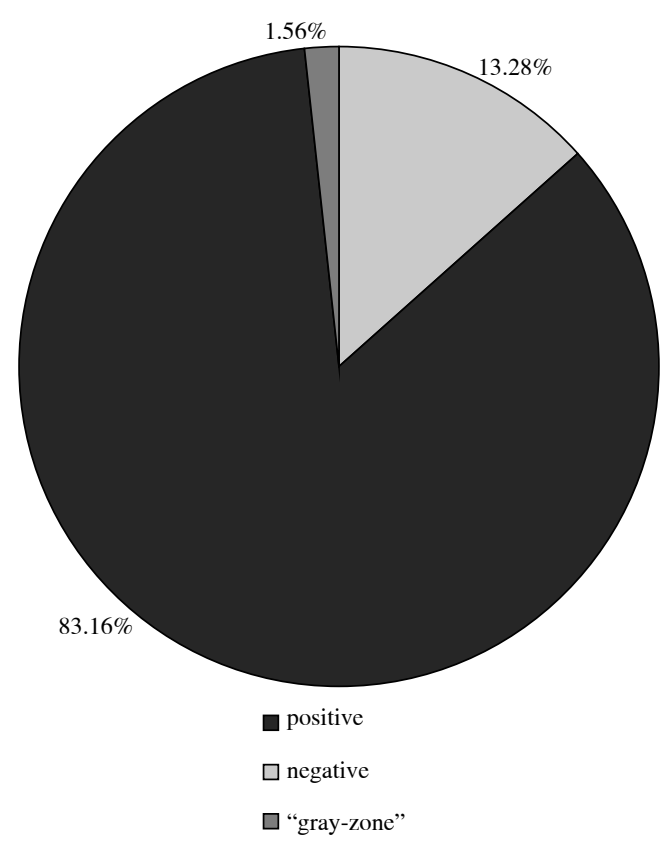

Fig. 1. Results of test detecting IgA antibodies against adenoviruses

Among girls aged 11-18 months $(n=28) 3$ positive results $(10.71 \%)$ were obtained, and there were no results in the grey zone. In the group of boys aged 11-18 months $(n=30)$, positive results for IgG antibodies against adenoviruses were obtained for 3 boys (10\%) and the results in a gray zone were obtained in two samples $(6.67 \%)$. In the age group of 19-27 months, in which there were 30 girls and 40 boys, four girls $(13.33 \%)$ were positive, and one in the grey zone $(3.33 \%)$. Positive results in the group of boys aged 19-27 months were achieved in 6 patients $(15 \%)$, while results in a grey zone were seen in 2 patients $(5 \%)$. No statistically significant difference in frequencies of positive results between the groups of girls aged 11-18 months and 19-27 months $(p=1.000)$ as well as between groups of boys aged 11-18 months and 19-27 months ( $p=$ $=0.7227$ ) were noted.

\section{The prevalence of IgA and IgG antibodies against adenoviruses}

Among all tested sera $(n=128), \operatorname{IgA}$ or IgG antibodies against adenoviruses were detected in 22 samples (17.19\%). Seventeen (13.28\%) sera were identified as IgA-positive and $16(12.50 \%)$ sera as IgG-positive. Eleven $(50 \%)$ sera were double positive (for IgA and IgG antibodies). Among the remaining positive samples for at least one class of examined immunoglobulins 3 sera were positive for $\operatorname{IgA}$ antibodies with the corresponding results in the grey zone of $\operatorname{IgG}, 3$ were positive for $\mathrm{IgA}$ with $\mathrm{IgG}$-negative results and 5 positive results for $\operatorname{IgG}$ with negative $\operatorname{IgA}$ (Fig. 3).

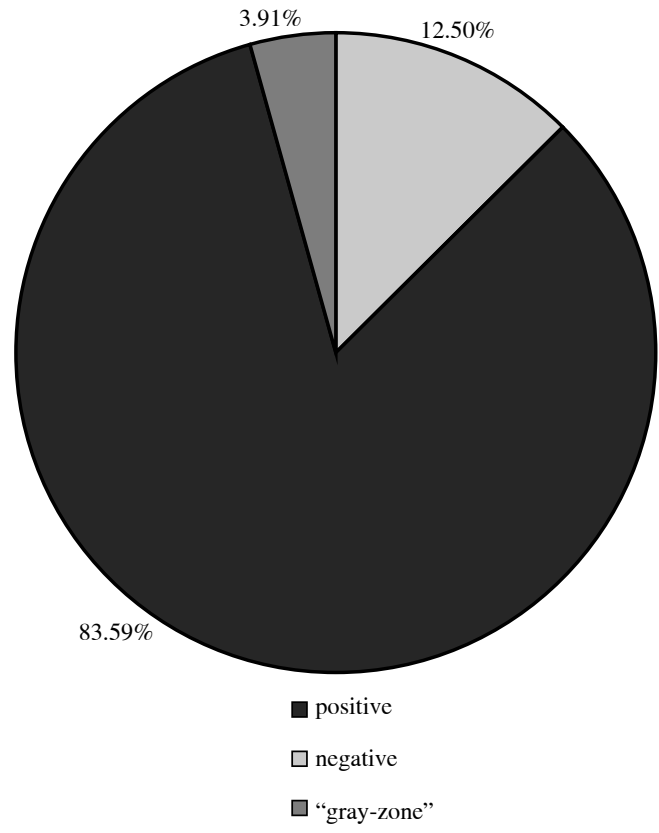

Fig. 2. Results of test detecting IgG antibodies against adenoviruses

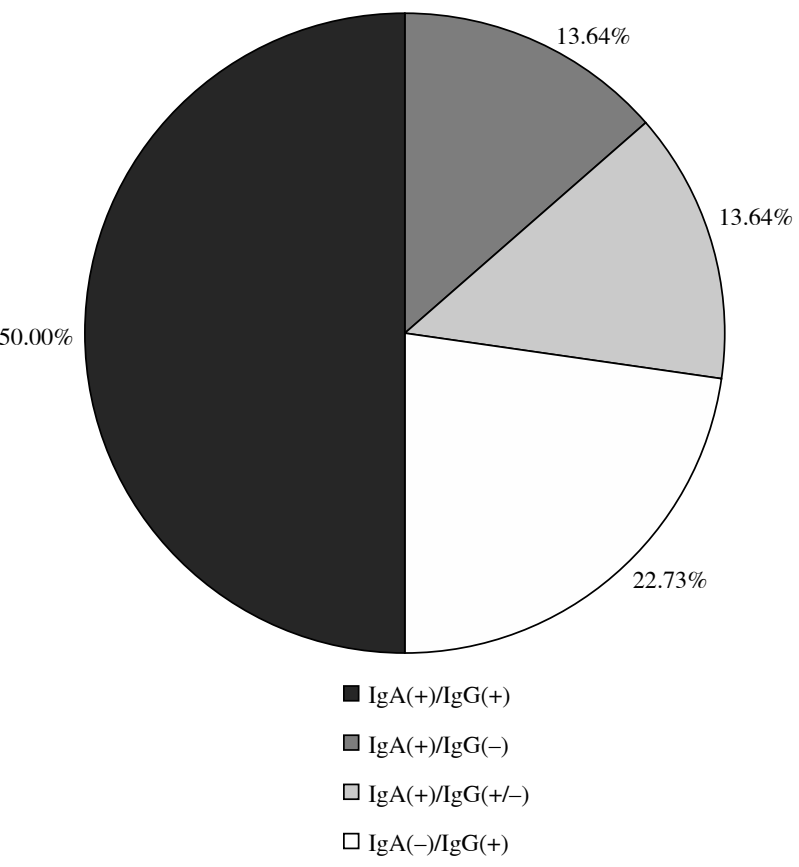

Fig. 3. The frequency of simple positive and double-positive samples of both tested antibodies

\section{Discussion}

Studies of the adenoviral infections published in the last twenty years have mainly focused on the detection of viruses in patients with symptoms of infection, or in 
immunosuppressed patients. More advanced analysis was performed to determine the serotype of the virus in the active phase of infection, using molecular biology methods [9-13]. Furthermore the possibility of using adenovirus as a gene therapy vector was studied [14].

There are no reports of the prevalence of specific antibodies against adenoviruses in the Polish population, to which our study could be referred. However, the study permits an initial estimation of adenovirus seropositivity among children.

In the present paper a low prevalence of antibodies to adenoviruses was found, both IgA and IgG. This result is inconsistent with the observations of Weinberg et al., regarding determination of antibodies in infants and children aged 1-24 months (median 7.28 months), which showed that $80 \%$ of children aged over 10 months were infected by adenovirus, as evidenced by the presence of $\mathrm{IgG}$ antibodies [15].

There was also no significant statistical difference comparing the positive results in the groups of girls and boys and in the age groups 11-18 and 19-27 months. This may be due to the low number of patients included to the study, as well as the small proportion of positive and negative results for both classes of antibodies. Additionally, it should be considered that the immunological system of the young children included to the study were not fully developed, which may be reflected in the decreased production of antibodies. An important factor influencing the small percentage of positive results may be individual differences in the ability to produce antibodies among the children enrolled in the study group. Based on our results, the need for further analysis is clear, including enlargement of the studied group and evaluation of anti-adenoviral IgM antibodies to distinguish patients in the active phase of primary infection. Since the study was conducted among hospital patients, to receive representative epidemiological data on the prevalence of anti-adenovirus antibodies in young children, a group of healthy children should be included in the study.

Among the patients who possessed adenoviral antibodies in at least one class, half of them (11/22) had a positive result in both $(\operatorname{Ig} \mathrm{A}$ and $\operatorname{IgG})$, which may suggest an active form of the infection. The authors cannot conclude that the cause of hospitalisation was adenoviral infection, because they do not have any clinical information about the patients. The study was in fact conducted purely in the laboratory, and epidemiological and personal data of the enrolled patients were not fully available.

Virion-Serion $\mathrm{GmbH}$, the manufacturer of commercial ELISA tests for the detection of $\operatorname{IgA}$ and $\mathrm{IgG}$ antibodies against adenoviruses, which are recommended for the diagnosis of acute adenoviral infections, presented reference ranges of the anti-adenoviral antibodies (either IgG or IgA) for healthy adults [16]. Immunoglobulin A and IgG antibodies against adenoviruses in serum of randomly selected blood donors from the area of Southern Germany were detected. For IgA antibodies $7.9 \%$ positive and $3.4 \%$ in grey zone results were obtained and for IgG positive results were not achieved and only $2.2 \%$ were seen in the grey zone. These values are lower than those obtained in the present study, probably due to the specificity of the test, dedicated to the detection of acute infections, the use of serum from healthy individuals and not from hospitalised patients, as in this study, as well as inclusion in the study of just adults. It was established that antibodies to adenoviral antigens disappear from circulation after about 10 years after the disappearance of the infection [16]. In addition, it is worth noting that children are much more exposed to viral infections than adults: therefore, a higher prevalence of specific antibodies among them can be expected.

On the basis of our results it can be concluded that the assessment of the occurrence of antibodies against adenoviruses does not coincide with epidemiological data on the spread of infections among young children. The tests used in the study may be more useful in distinguishing the active form of the infection from seropositivity due to prior infection.

It should also be noted that in this study a commercial ELISA test dedicated to the detection of acute adenoviral infection was used; thus they may have a limited value for the serological assessment of potentially healthy people. More detailed analysis of the usefulness of this type of testing for epidemiological purposes is also indicated.

\section{Authors declare no conflict of interest.}

\section{References}

1. Dzieciątkowski T, Rola A, Midak-Siewirska A (2008): Adenoviral infections in humans. Postepy Mikrobiologii 47: 15-22.

2. Murray P, Rosenthal KS, Pfaller MA (2012): Adenowirusy. In: Mikrobiologia. $6^{\text {th }}$ ed. Wydawnictwo Elsevier, Wroclaw 52: 491-499.

3. Stercz B, Perlstadt H, Nagy K, Ongrádi J (2013): Immunochemistry of adenoviruses: limitations and new horizons of gene therapy. Acta Microbiol Immunol Hung 60: 447-459.

4. Nadeau I, Kamen A (2003): Production of adenovirus vector for gene therapy. Biotechnol Adv 20: 475-489.

5. Stopa M, Dulak J, Józkowicz A (2007): The most important features of adenoviral vectors. Biotechnologia 3: 22-32.

6. Bauer U, Flunker G, Bruss K, et al. (2005): Detection of antibodies against adenovirus protein IX, fiber, and hexon in human sera by immunoblot assay. J Clin Microbiol 43: 4426-4433.

7. Rubicz R, Leach CT, Kraig E, et al. (2011): Seroprevalence of 13 common pathogens in a rapidly growing U.S. minority population: Mexican Americans from San Antonio, TX. BMC Research Notes 4: 433-442.

8. Kagami H, Atkinson JC, Michalek SM, et al. (1998): Repetitive adenovirus administration to the parotid gland: role of 
immunological barriers and induction of oral tolerance. Hum Gene Ther 9: 305-313.

9. Dey RS, Ghosh S, Chawla-Sarkar M, et al. (2011): Circulation of a novel pattern of infections by enteric adenovirus serotype 41 among children below 5 years of age in Kolkata, India. J Clin Microbiol 49: 500-505.

10. Green M (2002): Viral infections and pediatric liver transplantation. Pediatric Transplant 6: 20-24.

11. Michaels M, Green M, Wald ER, et al. (1992): Adenovirus infection in pediatric orthotopic liver transplant recipients. J Infect Dis 165: 170-174.

12. Rynans S, Dzieciątkowski T, Krenke R, et al. (2011): Real-time PCR assay for detection and quantification of human adenoviruses in patients with haematological malignancies and symptoms of lower respiratory tract infection. Przeglad Epidemiologiczny 65: 333-338 [Polish].

13. Zou L, Zhou J, Li H, et al. (2012): Human adenovirus infection in children with acute respiratory tract disease in Guangzhou, China. APMIS 120: 683-688.

14. Weinberg A, Fink MCDS, Takimoto S, et al. (1989): Enzyme linked immunosorbent assay: determination of anti-adenovirus antibodies in an infant population. Revista do Instituto de Medicina Tropical de Săo Paulo 31: 336-340.

15. Virion/Serion GmbH, SERION ELISA classic, Adenovirus IgA/IgG,http://www.virion-serion.de/download/gebrauchsanweisung/adenovirus/IFU-Adenovirus-SL.pdf

16. Flomenberg P (2012): Epidemiology and clinical manifestations of adenovirus infection. UpToDate. 